\title{
Morphology and Kinetics of Phase Separating Transparent Xanthan-Colloid Mixtures
}

\author{
Gijsberta H. Koenderink, ${ }^{*}{ }^{\dagger}$ Dirk G. A. L. Aarts, ${ }^{\dagger}$ Volkert W. A. de Villeneuve, ${ }^{\dagger}$ \\ Albert P. Philipse, ${ }^{\dagger}$ Remco Tuinier, ${ }^{\ddagger}$ and Henk N. W. Lekkerkerker ${ }^{\dagger}$ \\ Van't Hoff Laboratory for Physical and Colloid Chemistry, Debye Institute, Utrecht University, Padualaan 8, \\ $3584 \mathrm{CH}$ Utrecht, The Netherlands, and Institut für Festkörperforschung, Weiche Materie, \\ Forschungszentrum Jülich, D-52425 Jülich, Germany
}

Received August 7, 2002; Revised Manuscript Received October 17, 2002

\begin{abstract}
We present a study on the morphology and kinetics of depletion-induced phase separation in aqueous xanthan-colloid mixtures with light microscopy and small angle light scattering (SALS), using fluorinated colloids with a refractive index close to that of water to prevent complications of multiple scattering. Microscopy with the direction of observation perpendicular to gravity enabled us to observe the development of the microstructure during the entire phase separation process including the formation of a macroscopic interface. Bicontinuous structures typical of a spinodal decomposition mechanism were observed at early times. These structures coarsened in time until hydrodynamic flow resulted in lane formation. Close to the binodal, a nucleation-and-growth mechanism was observed with formation of droplets. The coarsening kinetics were studied in more detail with SALS and turbidity measurements. Above polysaccharide concentrations at which entanglements become dominant, a slower coarsening and macroscopic phase separation were found because of the high continuous phase viscosity.
\end{abstract}

\section{Introduction}

Polysaccharides are often used to prevent dispersed particles from creaming or sedimentation in water-based products such as salad dressings, sauces, and other food emulsions ${ }^{1,2}$ or suspensions of pesticide particles. ${ }^{3}$ The anionic polyelectrolyte xanthan is an especially efficient thickener because it strongly increases the continuous phase viscosity already at low concentrations (typically $0.5 \mathrm{wt} \%$ ). This is due to its high molar mass (order of $10^{3} \mathrm{~kg} / \mathrm{mol}$ ) and semirigid molecular structure (persistence length $120 \mathrm{~nm}$ ). It was noted in a number of experimental studies that addition of small amounts (typically $0.01 \mathrm{wt} \%$ ) of xanthan actually accelerates creaming or sedimentation. ${ }^{3-7}$ This effect was shown to be most likely due to depletion-induced attraction and consequent flocculation of the dispersed droplets or particles. Depletion attraction arises from the fact that for entropic reasons polymer chains avoid the space between two neighboring colloids, leading to an unbalanced osmotic pressure exerted by the polymers. ${ }^{8,9}$ Depletion attraction leads to colloid clustering and, at sufficiently high polymer concentrations, to macroscopic phase separation into a colloid-rich and colloid-poor phase. At higher xanthan concentrations $(\sim 0.2 \mathrm{wt} \%)$, sedimentation was delayed or even inhibited. ${ }^{1,3,5,7}$ This effect was ascribed to thickening and/or depletion-induced formation of a space-filling particle network of flocculated particles. Depletion effects therefore

* To whom correspondence should be addressed. E-mail: g.h.koenderink@ chem.uu.nl.

Utrecht University.

$\doteqdot$ Forschungszentrum Jülich. play an important role in controlling the long-term stability and texture of xanthan-containing products.

Accordingly, the effect of xanthan on the sedimentation rate of oil-in-water emulsions and particle suspensions has been extensively studied. Also, the rheology of the reversible particle gels formed at high particle and polymer concentrations has been investigated. ${ }^{3}$ However, so far studies of the mechanism of the phase separation process induced by xanthan are still lacking. A major obstacle is the large turbidity and concomitant multiple scattering of mixtures of xanthan and oil droplets or colloidal particles in water, which prevents the use of light scattering or optical microscopy. In this work, we solve this problem by using PFA (Teflon copolymerized with perfluoroalkylvinyl ether) spheres with a refractive index $n=1.36$, very close to that of water $(n=$ 1.33). Moreover, PFA particles are optically anisotropic, ${ }^{10,11}$ giving the interesting opportunity to study the hindered rotational and translational mobility of the colloids in xanthan solutions by depolarized dynamic light scattering (DDLS). The PFA spheres have a radius of $93 \mathrm{~nm}$, which is an order of magnitude smaller than the colloid size in many practical situations, such as suspensions of pesticide particles ${ }^{3}$ and oilin-water emulsions. ${ }^{4-6}$ Nevertheless, the use of the PFA particles allows us to investigate the xanthan-induced phase separation process in more detail and at high particle concentrations.

In the following, we present a systematic study of the phase separation morphology and kinetics for xanthan/colloid mixtures with xanthan concentrations between 0.03 and 0.20 wt $\%$, which is a range relevant to practical applications. ${ }^{1}$ The time evolution of the phase separation morphology is 
studied by turbidity measurements, microscopy, and small angle light scattering (SALS). The initial process of phase separation of a homogeneous mixture into two phases can in principle proceed through two distinct ways, namely, nucleation-and-growth (NG) or spinodal decomposition (SD), depending on the quench depth. For small quenches, that is, in the metastable region between the binodal and the spinodal, the system is stable to small concentration fluctuations unless they exceed a certain critical radius. Sufficiently large droplets will grow by Ostwald ripening or coalescence or both. For deeper quenches, that is, in the unstable region, concentration fluctuations grow spontaneously; one particular wavelength grows fastest, leading to a characteristic length scale in the structure. At early times, the resulting spinodal textures are typically bicontinuous, changing to droplet morphologies at later times. We will show that SALS data alone are insufficient to allow discrimination between SD and NG mechanisms but direct real-space observation with microscopy shows clear differences between SD and NG textures. In addition, microscopy allows us to study the late stages of phase separation, in which hydrodynamic flow becomes important.

\section{Experimental Section}

Xanthan Solutions. The xanthan (SKW Biosystems, $M$ $=4 \times 10^{6} \mathrm{~g} / \mathrm{mol}$ ), kindly provided by Dr. R. H. Tromp (Nizo, Ede, The Netherlands), was used without purification. Xanthan is a bacterial exo-polysaccharide, ${ }^{12}$ consisting of a cellullose backbone substituted on every second glucose residue with a trisaccharide side chain containing carboxylate anions. ${ }^{13}$ The rigid secondary structure (persistence length $q=120 \mathrm{~nm}^{14-16}$ ) is due to these bulky side chains. Most experimental evidence suggests that native xanthan forms wormlike double helices with a persistence length independent of temperature $\left(0-100{ }^{\circ} \mathrm{C}\right), \mathrm{pH}$, and electrolyte concentration (above $0.005 \mathrm{M}$ ). ${ }^{2}$ Assuming a linear mass density $M_{\mathrm{L}}=1945 \mathrm{~g} \mathrm{~nm}^{-1} \mathrm{~mol}^{-1},{ }^{17}$ the contour length, $L_{\mathrm{c}}$, is about $2 \mu \mathrm{m}$. The diameter of the xanthan double helix is ca. $2.2 \mathrm{~nm}$. The overlap concentration, $c_{\mathrm{p}}^{*}$, can be estimated from the molar weight, $M$, and radius of gyration, $R_{\mathrm{g}}$, using

$$
c_{\mathrm{p}}^{*}=3 M /\left(N_{\mathrm{av}} 4 \pi R_{\mathrm{g}}^{3}\right)
$$

Capron et al. ${ }^{18}$ reported $R_{\mathrm{g}}=155 \mathrm{~nm}$ (which would yield $c_{\mathrm{p}}^{*}$ $\cong 0.04 \mathrm{wt} \%$ ). However, this value seems to us unrealistically small given the large molecular weight and persistence length. Using the wormlike chain model of Benoit-Doty, ${ }^{19}$ which has earlier been successfully applied to xanthan, ${ }^{14,15,20}$ we calculate instead $R_{\mathrm{g}}=264 \mathrm{~nm}$. Throughout this paper, we will assume $R_{\mathrm{g}}=264 \mathrm{~nm}$ and, consequently, $c_{\mathrm{p}}^{*} \cong 0.008$ wt $\%$.

Xanthan was dissolved in water at $\mathrm{pH} 6$ in the presence of $0.1 \mathrm{M} \mathrm{NaCl}$ (to screen double-layer repulsions) and $2 \mathrm{mM}$ sodium azide (to prevent bacterial growth). The solution was heated to $85^{\circ} \mathrm{C}$ under stirring. When the temperature reached $85^{\circ} \mathrm{C}$, heating was stopped, and the solution was slowly cooled and stirred for another $15 \mathrm{~h}$. The viscosity of xanthan solutions as a function of xanthan concentration, $c_{\mathrm{p}}$, was

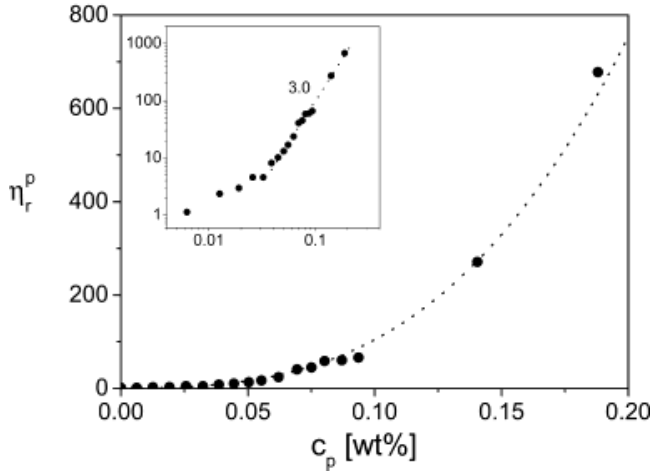

Figure 1. Reduced low-shear viscosity, $\eta_{r}^{p}$, of aqueous xanthan solutions $(0.1 \mathrm{M} \mathrm{NaCl})$ as a function of xanthan mass fraction, $c_{\mathrm{p}}$. Dotted line represents asymptotic power-law $\eta_{\mathrm{r}}^{\mathrm{p}} \propto c_{\mathrm{p}}{ }^{3}$ that fits the data well above $c_{p}=0.034 \mathrm{wt} \%$ (see double-logarithmic inset plot).

measured at $25.0^{\circ} \mathrm{C}$ using a Bohlin VOR rheometer equipped with a Couette geometry with outer radius $15.4 \mathrm{~mm}$ and gap width $1.4 \mathrm{~mm}$. The low-shear viscosity, $\eta_{\mathrm{r}}^{\mathrm{p}}$, normalized by the solvent viscosity $\left(\eta_{0}=0.866 \mathrm{mPa} \mathrm{s}\right)$, was determined by extrapolation of viscosity versus shear rate, $\dot{\gamma}$, curves to $\dot{\gamma}=0 \mathrm{~s}^{-1}$. Figure 1 shows that $\eta_{\mathrm{r}}^{\mathrm{p}}$ increases strongly with xanthan concentration $c_{\mathrm{p}}$. Above $0.034 \mathrm{wt} \%\left(4 c_{\mathrm{p}}^{*}\right)$, we find a power-law dependence $\eta_{\mathrm{r}}^{\mathrm{p}} \propto c_{\mathrm{p}}{ }^{k}$ with $\kappa=3.0$ (see inset), in fair agreement with theories for long entangled chains, ${ }^{21,22}$ which predict $\kappa=3.5$, and with previous experimental findings. ${ }^{23}$

PFA Dispersions. The colloidal PFA spheres (Hyflon) with a radius $R_{\mathrm{c}}$ of $93 \mathrm{~nm}$ were a kind gift from Ausimont S.p.A. (Milan, Italy). The particles are prepared by dispersion polymerization in the presence of an anionic surfactant. ${ }^{10}$ The original latex was purified by dialyzing for several weeks against flowing demineralized water. The particles are electrostatically stabilized by a negative charge due to surface carboxyl groups (zeta potential $-30 \mathrm{mV}$ at $\mathrm{pH}$ 5). ${ }^{11}$

Phase Behavior. The colloid-polymer phase diagram was constructed by visual inspection of xanthan/PFA mixtures with different xanthan concentrations, $c_{\mathrm{p}}$, and colloid concentrations, $c_{\mathrm{c}}$, after homogenization by vigorous shaking. Any air bubbles were removed by short $(<1 \mathrm{~s})$ ultrasonication or by centrifugation. Observations were made at room temperature $\left(23^{\circ} \mathrm{C}\right)$. Volumes of coexisting phases were measured with an altimeter to locate the critical point.

Turbidity. Turbidity measurements were performed using a home-built optical multiple channel analyzer (OMA) with a randomly polarized $\mathrm{He}-\mathrm{Ne}$ laser $(5 \mathrm{~mW}$, wavelength $\lambda=$ $632.8 \mathrm{~nm}$ ) as the light source. ${ }^{24}$ The path length $d$ of the optical glass cuvettes was $1 \mathrm{~mm}$. The transmitted light intensity was monitored over a period of $1 \mathrm{~h}$.

Light Microscopy. Light microscopy was done with a Nikon Eclipse 400 Pol microscope in transmission equipped with Nikon Plan Fluor objectives of $2 \times, 10 \times, 20 \times$, and $40 \times$ (the latter two are extra-long working distance objectives). The light microscope was placed horizontally such that the light path was perpendicular to the earth gravitational field enabling us to observe the samples sideways and hence see the complete time evolution of phase separation from initial to final state. A Sony XC-8500-CE video camera (CCD camera) was attached to the microscope and coupled to a 
desktop computer. A maximum of 50 images per second could be captured with a resolution of $72 \mathrm{dpi}$ and a size of 768 pixels $\times 576$ pixels. Samples were prepared in optical glass cuvettes (path length $1 \mathrm{~mm}$ ) and were quickly homogenized. Defining time $t=0$ as the moment at which homogenization was stopped, we took the first images after about $5 \mathrm{~s}$.

Small Angle Light Scattering. SALS measurements were done using a home-built setup ${ }^{25}$ with a randomly polarized $\mathrm{He}-\mathrm{Ne}$ laser $(5 \mathrm{~mW}, \lambda=632.8 \mathrm{~nm})$ as the light source. The scattered light was projected onto a screen with a hole in the center functioning as a beam stop. The intensity pattern was recorded by a 165 pixels $\times 192$ pixels CCD camera (Electrim, Princeton, NJ) using exposure times ranging from $400 \mathrm{~ms}$ for high $c_{\mathrm{c}}$ to $1000 \mathrm{~ms}$ for low $c_{\mathrm{c}}$. Typically 65 images were collected over a period of $1 \mathrm{~h}$. Time series were corrected for dark current and stray light by subtracting an early image. The scattering patterns were radially averaged to give plots of intensity $I(k, t)$ for wavevectors $k$ between 200 and $1500 \mathrm{~mm}^{-1}$. Here, $k$ is defined as $k=(4 \pi n / \lambda) \sin -$ $(\theta / 2)$ with $n$ being the solvent refractive index and $\theta$ the scattering angle. We note that at these small angles the particle form factor equals unity for colloidal particles and $I(k, t)$ is proportional to the static structure factor. Defining time $t=0$ as the moment at which homogenization (shaking) was stopped, we took the first measurements after $15 \mathrm{~s}$. The reproducibility of homogenization was checked by performing consecutive SALS measurements on some samples, giving highly reproducible results.

Depolarized Dynamic Light Scattering. The PFA particles are optically anisotropic, which permits simultaneous measurement of rotational and translational diffusion coefficients $D^{\mathrm{r}}$ and $D^{\mathrm{t}}$, by depolarized dynamic light scattering (DDLS). ${ }^{11}$ DDLS was done at $25{ }^{\circ} \mathrm{C}$ using vertically polarized light from an argon laser $(\lambda=514.5 \mathrm{~nm})$. The horizontally polarized scattered intensity, $I_{\mathrm{VH}}$, measured in the horizontal scattering plane, was selected with a GlanThompson polarizer placed in front of the photomultiplier. The normalized intensity autocorrelation functions $g_{\mathrm{VH}}(k, t)$ were fitted to a cumulant expression, $g_{\mathrm{VH}}(k, t)=\alpha+\beta$ exp$\left(-2\left(\Gamma(k) t+c(k) t^{2}\right)\right)$, with fitting parameters $\alpha, \beta, c(k)$, and $\Gamma(k)=12 D^{\mathrm{r}}+2 D^{\mathrm{t}}$. At infinite dilution, $D^{\mathrm{r}}$ and $D^{\mathrm{t}}$ are given by the Stokes-Einstein formulas $D_{0}^{\mathrm{t}}=k_{\mathrm{B}} T /\left(6 \pi \eta_{0} R\right)$ and $D_{0}^{\mathrm{r}}$ $=k_{\mathrm{B}} T /\left(8 \pi \eta_{0} R^{3}\right)$. In xanthan-PFA mixtures, depolarized scattering by xanthan is negligible.

\section{Results and Discussion}

Phase Behavior. Figure 2 shows the experimental phase diagram of aqueous mixtures of PFA spheres and xanthan with $0.1 \mathrm{M} \mathrm{NaCl}$ determined by visual inspection. Colloid and polymer concentrations, $c_{\mathrm{c}}$ and $c_{\mathrm{p}}$, respectively, are given in terms of weight percentages. The phase diagram was constructed along the dilution lines $1-10$, indicated by the solid lines, by determining the concentrations above which phase separation took place. The dashed curve represents the approximate location of the binodal. In addition, the estimated location of the critical point is shown $(\oplus$-symbol), determined from the position on the binodal where the

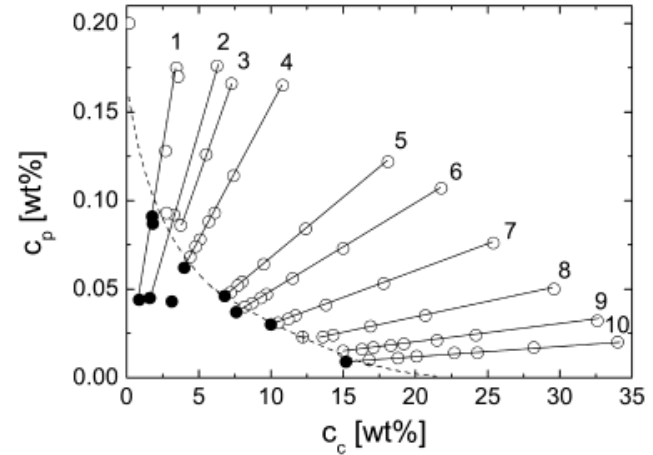

Figure 2. Phase diagram of xanthan/PFA mixtures in terms of weight fractions of colloid, $c_{\mathrm{c}}$, and polymer, $c_{\mathrm{p}}$. The dashed line shows the approximate location of the binodal with stable mixtures to the left (closed symbols) and phase-separating mixtures to the right (open symbols). Drawn, numbered lines indicate various dilution lines. The critical point is indicated by $\oplus$.

volumes of the coexisting phases were equal. A rough estimate of the depletion attraction, $W_{\text {dep }}\left(r=2 R_{\mathrm{c}}\right)$, at contact at the critical point $\left(c_{\mathrm{p}}=0.023 \mathrm{wt} \%\right)$ can be made using a scaling prediction by De Gennes ${ }^{26}$ valid for colloids in semidilute polymer solutions under good solvent conditions:

$$
W_{\text {dep }}\left(r=2 R_{\mathrm{c}}\right) \cong-k_{\mathrm{B}} T\left(\frac{R_{\mathrm{c}}}{\xi}\right)
$$

Using, in addition, a scaling relation for the correlation length, ${ }^{27,28} \xi \cong R_{\mathrm{g}}\left(c_{\mathrm{p}} / c_{\mathrm{p}}^{*}\right)^{-0.75}$, we obtain a contact potential of ca. $0.8 k_{\mathrm{B}} T$. For long-range potentials, such an attraction should indeed be sufficient to give liquid-gas phase separation. $^{29}$ It is yet unknown what the effect is of polymer semiflexibility on depletion interaction and the resulting binodals.

Below the binodal (filled symbols), mixtures did not phase separate but slowly sedimented under gravity giving amorphous sediments. Sedimentation was slower with increasing $c_{\mathrm{p}}$ because of the strongly increasing continuous phase viscosity (cf. Figure 1) caused by entangling of xanthan molecules; for concentrations $c_{\mathrm{p}} \geq 4 c_{\mathrm{p}}^{*}$, the viscosity increases as $\eta_{\mathrm{r}}^{\mathrm{p}} \propto c_{\mathrm{p}}{ }^{3}$.

Above the binodal (open symbols), mixtures became turbid within minutes after homogenization, and the turbidity increased over time, indicating the development of colloidrich and colloid-poor domains leading to enhanced scattering of light. The mixtures phase separated over time scales varying from minutes to hours, depending on their composition, into a colloid-rich amorphous bottom phase and a colloid-poor upper phase. The interface between the two phases was sharp and fluid, indicating a colloidal gas-liquid coexistence. Because of the significant density mismatch of $1 \mathrm{mg} / \mathrm{mL}$ between particles and solvent, we also observed sedimentation effects, complicating the determination of the binodal. Along dilution lines 8 and 9, close to the binodal, we could not distinguish between sedimentation and phase separation.

Close to the critical point $(\bigoplus$-symbol in Figure 2$)$, phase separation was slow, taking up to $8 \mathrm{~h}$ as seen by visual inspection of the interface forming between the colloidal gas and liquid phase. Following the dilution lines numbered 5 


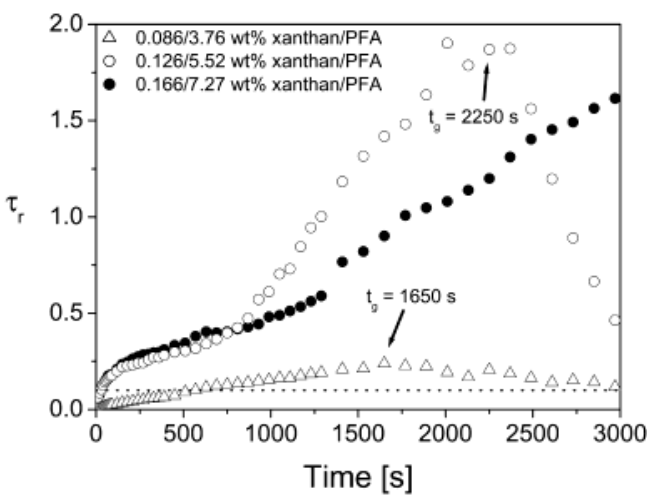

Figure 3. Time evolution of the turbidity for mixtures with a fixed $c_{\mathrm{c}} / c_{\mathrm{p}}$ ratio of 44 . The dotted line indicates $\tau_{\mathrm{r}}=0.1$. The two lowestconcentration samples start sedimenting after $t_{\mathrm{g}}=1650$ and $2250 \mathrm{~s}$. The third sample does not sediment within $3090 \mathrm{~s}$.

through 10 in the direction of increasing $c_{\mathrm{c}}$ and $c_{\mathrm{p}}$, phase separation became progressively faster. For the highest colloid and polymer concentrations, phase separation was completed within $30 \mathrm{~min}$. Somewhat different behavior was seen along dilution lines $1-4$. Upon increase of $c_{\mathrm{p}}$, phase separation first became faster. However, for $c_{\mathrm{p}}>0.15 \mathrm{wt}$ $\%$, macroscopic phase separation became slower, taking at least $12 \mathrm{~h}$ for dilution lines 1 and 2 . The latter mixtures did still become turbid within several minutes, and the turbidity increased over time (see also Figure 3). This nonmonotonic $c_{\mathrm{p}}$ dependence of macroscopic phase separation can be rationalized by a competition between the increasing continuous phase viscosity due to strong overlap of xanthan chains and the increasing attraction between particles due to an enhanced osmotic pressure of the xanthan solution. The increasing viscosity decreases particle mobilities and thereby retards the rate of macroscopic phase separation, while the enhanced attraction between particles promotes particle clustering and consequent macroscopic phase separation. Apparently, at intermediate xanthan concentrations, the increased attraction more than compensates for the increase in the viscosity, while at high $(>0.12$ wt $\%)$ xanthan concentrations, the viscosity prevents the particles or clusters from moving over large distances. Similar observations have been made for oil-in-water emulsions containing xanthan, in which droplet creaming was enhanced by xanthan concentrations between 0.05 and $0.25 \mathrm{wt} \%$ and delayed or prevented at xanthan concentrations above 0.25 wt $\% .^{1,4-6}$ Because of the large droplet sizes in these studies (order of $1 \mu \mathrm{m}$ ), the range of attraction is much shorter than that in the xanthan-PFA system, leading to an additional mechanism for delayed creaming, namely formation of transient particle gels, which temporarily arrest phase separation. In colloid-polymer mixtures with $R_{\mathrm{g}} / R \leq 0.2$, the polymer induces aggregation of the colloids in the colloid-rich phase, which, at sufficiently large polymer and colloid concentrations, leads to a space-spanning particle network. ${ }^{1,4,30-35}$ The reversible nature of the depletion-induced particle bonds allows restructuring of the network until the gel collapses, permitting macroscopic phase separation.

Both the turbidity increase and the macroscopic phase separation were reversible and reproducible for all samples. When a turbid or phase-separated sample was shaken, a clear and homogeneous solution was obtained. On standing, the dispersions became turbid again. The turbidity increase is caused by growing density inhomogenities related to particle clustering. Shaking the sample shear-melts these inhomogeneities. Apparently, particle clustering is reversible, as one would expect for depletion-induced attraction. Irreversible flocculation would manifest bridging attraction, which occurs when the polymer adsorbs onto the particles. In our samples, adsorption apparently does not occur, which is compatible with the fact that both xanthan and PFA are negatively charged. Depolarized dynamic light scattering (DDLS) confirmed that xanthan does not adsorb onto PFA. Any adsorption of xanthan on PFA, even at small xanthan additions, would give a significant (3-fold) increase of the radius of the PFA particles. Especially the rotational diffusion coefficient, which is inversely proportional to the particle volume, should be very sensitive to any adsorption of xanthan. We found that self-diffusion of the PFA spheres (results not shown) was hardly affected by xanthan at low $c_{\mathrm{p}}$, confirming that xanthan is nonadsorbing toward PFA.

The fact that we observe liquid-gas phase separation is in agreement with theoretical predictions for hard spheres with added polymer. The topology of the phase diagram for hard spheres with an added depletion agent only depends on the ratio $\zeta$ between the size of the polymer $\left(\sim R_{\mathrm{g}}\right)$ and that of the colloid $\left(R_{\mathrm{c}}\right){ }^{36-40}$ The range of the attraction is proportional to $R_{\mathrm{g}}$. For attractions of relatively long range $(\zeta>0.3)$, gas, liquid, and solid phases may coexist, while for shorter-ranged attractions $(\xi<0.3)$, the polymer only expands the fluid-solid coexistence region. In our case, the ratio $R_{\mathrm{g}} / R_{\mathrm{c}}$ equals 2.8 , so liquid-gas phase separation is indeed expected.

Turbidity. The turbidity increase with time observed for phase-separating mixtures was quantified by monitoring the transmitted light intensity over a period of $1 \mathrm{~h}$ after sample homogenization. Figure 3 shows the time evolution of the relative turbidity, $\tau_{\mathrm{r}}$, defined as

$$
\tau_{\mathrm{r}}=\ln \left(\frac{I_{\mathrm{t}}(t=0)}{I_{\mathrm{t}}(t)}\right)
$$

for three mixtures with a fixed $c_{\mathrm{c}} / c_{\mathrm{p}}$ ratio of 44 (dilution line $3)$. In eq $2, I_{\mathrm{t}}(t=0)$ and $I_{\mathrm{t}}(t)$ represent the transmitted light intensities at time $t=0$ and time $t$, respectively. For the more concentrated samples $\left(c_{\mathrm{p}}=0.126, c_{\mathrm{c}}=5.52\right.$ and $c_{\mathrm{p}}=$ $\left.0.166, c_{\mathrm{c}}=7.27 \mathrm{wt} \%\right)$, a stronger increase of the turbidity was found than for the more dilute sample $\left(c_{\mathrm{p}}=0.086, c_{\mathrm{c}}\right.$ $=3.76 \mathrm{wt} \%$ ). After a certain time (which we label $t_{\mathrm{g}}$ ), gravity-induced sedimentation of colloid-rich domains and creaming of colloid-poor domains caused the turbidity to drop (indicated by arrows in Figure 3). For the two more dilute samples in Figure 3, this happened after $t_{\mathrm{g}}=1650$ and $2250 \mathrm{~s}$, while for the most concentrated sample, there was no evidence of sedimentation within $3090 \mathrm{~s}$. Table 1 contains experimental values for $t_{\mathrm{g}}$ for all samples studied (i.e., mostly close to the dilution lines 1-4 in Figure 2). On comparison of the data, it can be seen that sedimentation of colloid-rich clusters is retarded by increasing $c_{\mathrm{p}}$ and accelerated by increasing $c_{\mathrm{c}}$. 
Table 1. Summary of Turbidity and SALS Results

\begin{tabular}{|c|c|c|c|c|c|c|c|c|}
\hline \multicolumn{3}{|c|}{ sample } & \multicolumn{2}{|c|}{ turbidity } & \multicolumn{4}{|c|}{ SALS } \\
\hline$c_{C} / c_{p}^{a}$ & $c_{p}[w t \%]$ & $c_{\mathrm{c}}[\mathrm{wt} \%]$ & $t_{10}[\mathrm{~s}]$ & $\operatorname{tg}[\mathrm{s}]$ & $\varphi$ & $\psi$ & $\psi / \varphi$ & $\Lambda(t=0.8 \mathrm{~s})$ \\
\hline $\begin{array}{l}21 \\
21 \\
20\end{array}$ & $\begin{array}{l}0.128 \\
0.170 \\
0.175\end{array}$ & $\begin{array}{l}2.69 \\
3.57 \\
3.45\end{array}$ & $\begin{array}{l}416 \\
440\end{array}$ & $\begin{array}{l}>3090 \\
>3090\end{array}$ & $\begin{array}{l}0.11 \\
0.063 \\
0.11\end{array}$ & $\begin{array}{l}0.31 \\
0.31 \\
0.30\end{array}$ & $\begin{array}{l}2.8 \\
4.9 \\
2.8\end{array}$ & $\begin{array}{l}13.6 \\
10.9 \\
12.5\end{array}$ \\
\hline $\begin{array}{l}36^{b} \\
36\end{array}$ & $\begin{array}{l}0.092 \\
0.176\end{array}$ & $\begin{array}{l}3.28 \\
6.27\end{array}$ & & & $\begin{array}{l}0.16 \\
0.13\end{array}$ & $\begin{array}{l}0.33 \\
0.33\end{array}$ & $\begin{array}{l}2.0 \\
2.5\end{array}$ & $\begin{array}{c}19.3 \\
9.38\end{array}$ \\
\hline $\begin{array}{l}44^{b} \\
44 \\
44\end{array}$ & $\begin{array}{l}0.086 \\
0.126 \\
0.166\end{array}$ & $\begin{array}{l}3.76 \\
5.52 \\
7.27\end{array}$ & $\begin{array}{r}542 \\
24 \\
23\end{array}$ & $\begin{array}{r}1650 \\
2250 \\
>3090\end{array}$ & $\begin{array}{l}0.15 \\
0.47\end{array}$ & $\begin{array}{l}0.35 \\
0.069\end{array}$ & $\begin{array}{l}2.4 \\
0.15\end{array}$ & $\begin{array}{l}20.0 \\
17.1\end{array}$ \\
\hline $\begin{array}{l}72 \\
72\end{array}$ & $\begin{array}{l}0.085 \\
0.170\end{array}$ & $\begin{array}{c}6.14 \\
12.3\end{array}$ & & & $\begin{array}{l}0.45 \\
0.12\end{array}$ & $\begin{array}{l}0.060 \\
0.10\end{array}$ & $\begin{array}{l}0.13 \\
0.81\end{array}$ & $\begin{array}{l}14.0 \\
12.5\end{array}$ \\
\hline 86 & 0.145 & 12.5 & 1.72 & 150 & & & & \\
\hline 521 & 0.038 & 19.8 & 13 & 70 & & & & \\
\hline
\end{tabular}

a The samples with $c_{\mathrm{c}} / c_{\mathrm{p}}=20,36,44$, and 72 correspond to dilution lines $1,2,3$, and 4 , respectively. ${ }^{b}$ Samples that demixed by nucleationand-growth.
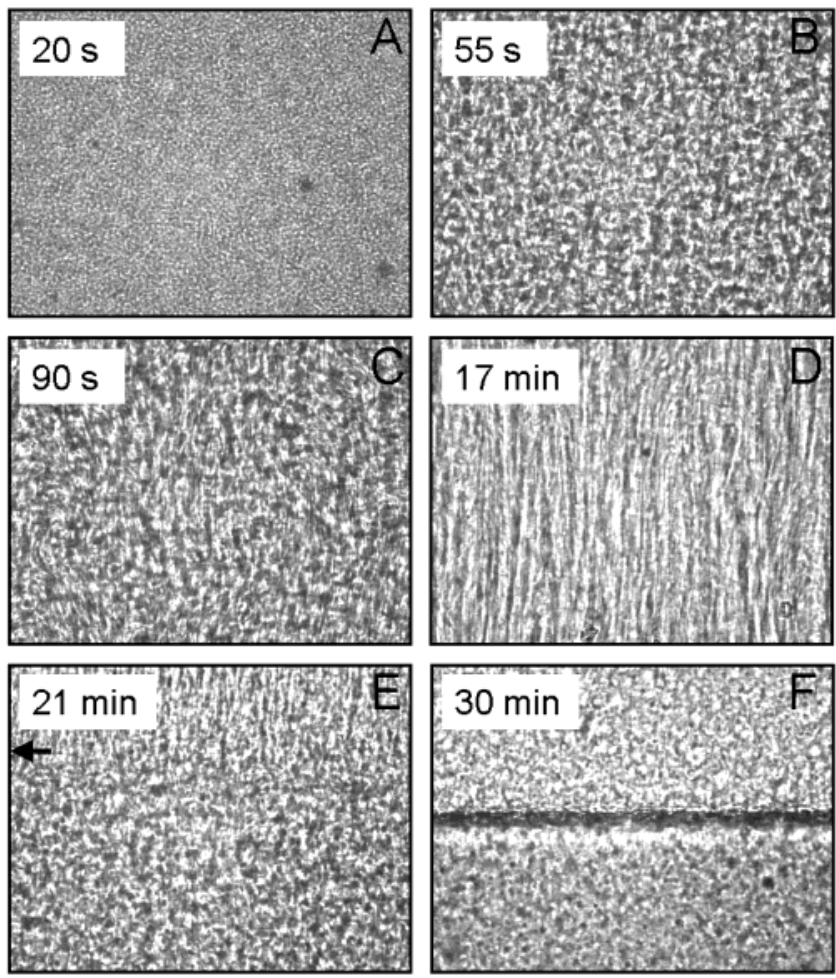

Figure 4. Optical micrographs of a phase-separating xanthan/PFA mixture $\left(c_{p}=0.021, c_{c}=26.6 \mathrm{wt} \%\right)$ taken at various times after homogenization, showing textures typical of spinodal decomposition. The images show a region measuring $706 \mu \mathrm{m}$ by $530 \mu \mathrm{m}$.

The rate of increase of the turbidity is a measure of the coarsening kinetics. A convenient, though somewhat arbitrary, measure of this rate is the time $t_{10}$ at which the transmitted intensity is reduced to $10 \%$ of its value at time $t=0\left(\tau_{\mathrm{r}}\left(t_{10}\right)=0.1\right)$. As seen by inspection of Table 1 , raising $c_{\mathrm{c}}$ at fixed $c_{\mathrm{p}}$ strongly decreases $t_{10}$. Likewise, raising $c_{\mathrm{p}}$ in the region $c_{\mathrm{p}}=0.04-0.12 \mathrm{wt} \%$ decreases $t_{10}$. On a final note, for all samples studied, the turbidity increased immediately after homogenization without any (observable) induction time.

Optical Microscopy. Real-space impressions of the time evolution of the microscopic structure of phase-separating mixtures were obtained with optical microscopy. Figure $4 \mathrm{~A}-\mathrm{F}$ shows a typical time evolution for a sample quenched deep in the two-phase region of the phase diagram with high
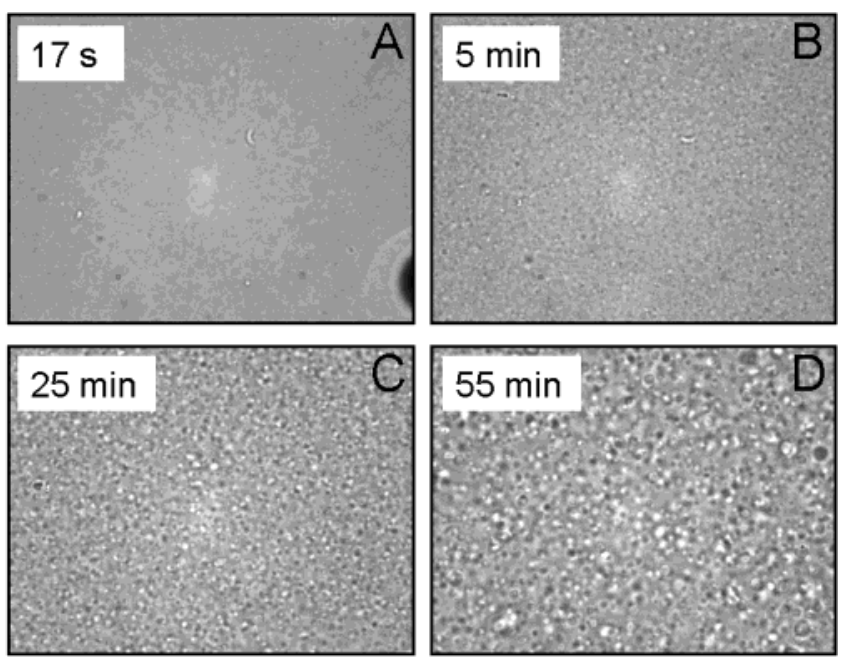

Figure 5. Optical micrographs of a phase-separating xanthan/PFA mixture $\left(c_{\mathrm{p}}=0.099, c_{\mathrm{c}}=3 \mathrm{wt} \%\right)$ taken at various times after homogenization, showing features typical of a nucleation-and-growth process. The images show a region measuring $350 \mu \mathrm{m}$ by $270 \mu \mathrm{m}$.

$c_{\mathrm{c}}$ and moderate $c_{\mathrm{p}}\left(c_{\mathrm{p}}=0.021, c_{\mathrm{c}}=26.6 \mathrm{wt} \%\right)$. Figure $4 \mathrm{~A}$, taken $20 \mathrm{~s}$ after sample homogenization, shows a bicontinuous structure of darker and lighter domains (with an average size of $\sim 20$ particle diameters) typical of spinodally decomposing structures. Interfaces between the domains became visibly sharper only $35 \mathrm{~s}$ later (Figure 4B) as a result of coarsening. Restructuring of the interfaces and coalescence of domains resulted in larger structures until at a size of $\sim 60$ particle diameters gravity led to hydrodynamic flow, pulling the phases apart (Figure 4C, $t=90 \mathrm{~s}$ ). In the upper part of the cuvette, in what later became the colloidal gas phase, hydrodynamic flow led after about $17 \mathrm{~min}$ to the formation of lanes of the lighter phase moving upward and lanes of the heavier phase moving downward (Figure 4D). The lanes were on average 45 particle diameters wide. After about $35 \mathrm{~min}$, the lanes broke up into spherical droplets. A rough interface between colloidal gas and liquid phase was meanwhile already forming, as indicated by the arrow in Figure $4 \mathrm{E}(t=21 \mathrm{~min})$. Above the interface, lanes were still present, while below the interface in the colloidal liquid phase, no lanes were seen. In time, the macroscopic interface sharpened (Figure 4F, $t=30 \mathrm{~min}$ ). Visual inspection with the naked eye suggested that for this particular sample phase separation was complete about $1 \mathrm{~h}$ after homogenization, although microscopy revealed that both phases still contained small droplets of the other phase, which slowly moved either up- or downward to their respective bulk phases. Thus, it is difficult to define exactly when phase separation is complete. In the upper-left corner of the two-phase region of the phase diagram, at lower $c_{\mathrm{c}}$ and higher $c_{\mathrm{p}}$, similar spinodal textures were seen at early times as that shown in Figure 4A. Note that we did not study the late-stage coarsening and hydrodynamic flows in this region.

For concentrations $c_{\mathrm{p}} \approx 0.1$ and $c_{\mathrm{c}} \approx 3 \mathrm{wt} \%$, that is, close to the binodal, we observed a completely different morphology from that shown in Figure 4, namely, one of isolated droplets. After homogenization, initially no or hardly any structure was observed (see Figure 5A, $t=17 \mathrm{~s}$ ). After about $40 \mathrm{~s}$, the first droplets of the heavy phase became visible 
$0.76 \mathrm{~s}$

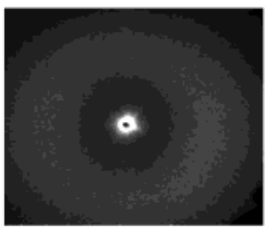

$330 \mathrm{~s}$

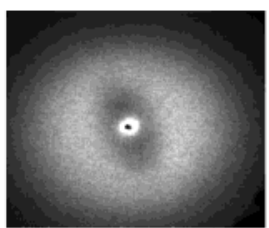

$12.14 \mathrm{~s}$

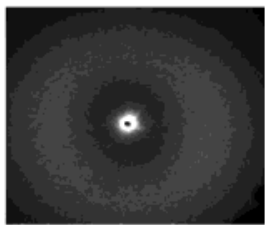

$868 \mathrm{~s}$

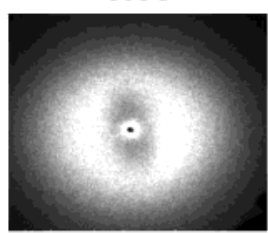

$43.05 \mathrm{~s}$

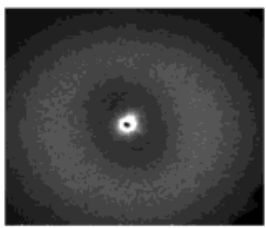

$2262 \mathrm{~s}$

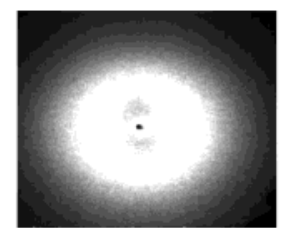

$136 \mathrm{~s}$

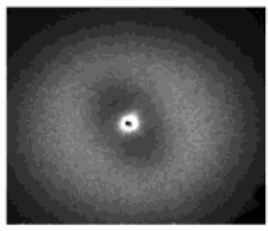

$3315 \mathrm{~s}$

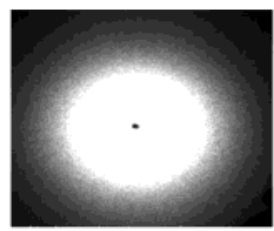

Figure 6. Scattering profiles of a phase-separating mixture with $c_{\mathrm{c}}=6.3$ and $c_{\mathrm{p}}=0.18 \mathrm{wt} \%$ as projected on a screen and recorded by a CCD camera. After ca. $30 \mathrm{~min}$, sedimentation distorts the SALS ring.

with sizes of about 10 particle diameters, and after $3 \mathrm{~min}$, many droplets were visible, as shown in Figure 5B $(t=5$ $\mathrm{min})$. Both the presence of an initiation time and the morphology of single droplets strongly point to a nucleationand-growth mechanism. When the sample was shaken and observed again, the initiation time was not always present, probably because of incomplete homogenization. The droplets increased in size over time, reaching a size of about 20 particle diameters after $25 \mathrm{~min}$ (Figure 5C). We did not observe any coalescence of droplets, which leads us to assume that the growth process is Ostwald ripening. After about $35 \mathrm{~min}$, the size of the individual droplets was such that gravity pulled them down and they no longer visibly exhibited Brownian motion. From this point on, the droplets became more polydisperse in size, as illustrated by Figure $5 \mathrm{D}(t=55 \mathrm{~min})$. Furthermore, large droplets were no longer spherical but deformed into tearlike shapes. After about 3 $\mathrm{h}$, phase separation was complete.

We note that the spinodal is likely to be very close to the binodal for the xanthan-PFA mixtures because of the longrange nature of the attraction, ${ }^{41}$ so nucleation-and-growth probably occurs only in a narrow part of the phase diagram. In future work, this speculation could be confirmed by measuring the location of the spinodal, for instance, by measuring the apparent colloid diffusion coefficient or the correlation length in the stable region of the phase diagram. ${ }^{42,43}$

Small Angle Light Scattering. Using small angle light scattering (SALS), we analyzed various mixtures with compositions corresponding to dilution lines 1-4 (for higher $c_{\mathrm{c}}$ multiple scattering occurred because of incomplete refractive index matching of the colloids). Figure 6 shows a typical time series of scattering patterns for a mixture with $c_{\mathrm{c}}=$ 6.27 and $c_{\mathrm{p}}=0.176 \mathrm{wt} \%$ (dilution line 2). Already in the first measurement, $15 \mathrm{~s}$ after homogenization, a SALS ring is seen, indicating that a dominant density fluctuation has (almost) instantly developed. ${ }^{44}$ The ring continuously moved to smaller angles and became brighter with time. In the late stage of the phase separation process, the circular ring changed into an ellipsoidal shape (after 30 min in Figure 6), likely due to gravity-induced deformation of droplets or domains. Possibly, lane formation as seen with microscopy for samples close to dilution lines 8 and 9 (Figure 4D) affects

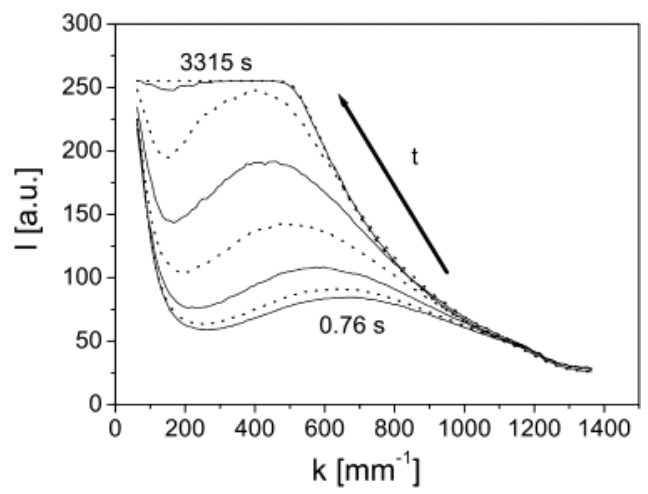

Figure 7. Time series of SALS patterns $l(k)$ obtained by radial averaging of the scattering profiles in Figure 6 . Over time, the intensity peak grows in intensity, $I_{\max }$, and shifts to smaller wavevector, $k_{\max }$. After ca. $30 \mathrm{~min}, I$ at small $k$ exceeds the detection limit. The curves are alternately drawn and dotted for clarity.

the scattering patterns. The scattering data after radial averaging are shown in Figure 7; the rings in Figure 6 translate to peaks in the SALS patterns. The peak position, which we label by $k_{\max }$, shifts to smaller wavevectors, and the maximum scattered intensity, labeled $I_{\max }$, increases in time. This indicates a coarsening of the characteristic length scale, $\Lambda_{\max }=2 \pi / k_{\max }$, and growth of the amplitude of the density fluctuations, in agreement with the features seen with microscopy (Figure 4). In the large- $k$ limit $\left(k \gg k_{\max }\right)$, the scattered intensity scales as $I \propto k^{-4}$ in accordance with Porod's law ${ }^{45}$ expected for interfaces between coexisting domains, which are sharp compared to the wavelength of light.

For all samples studied, the time evolution of the SALS patterns was qualitatively similar: a peak appeared already in the first measurement, and the peak position decreased and the peak height increased from the onset of the experiment. The first recorded value of the peak position (at $t=0.8 \mathrm{~s}$ ) should reflect the initial characteristic length scale, $\Lambda_{\max }(t=0)$, of the fastest-growing density variation. As shown in Table 1 , values for $\Lambda_{\max }(0.8 \mathrm{~s})$ vary between 9 and $20 \mu \mathrm{m}$, that is, between 47 and 105 sphere diameters. Further, the data suggest that $\Lambda_{\max }$ is (slightly) smaller with deeper quenches into the two-phase region of the phase diagram. The length scales are comparable with the length scales of domains or droplets found with microscopy in the 

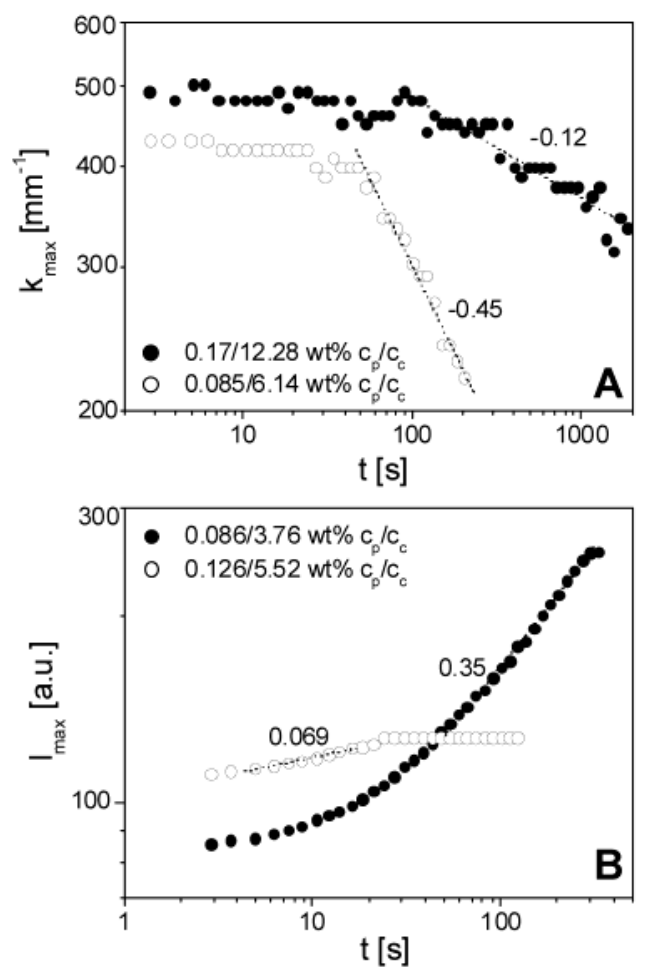

Figure 8. Double-logarithmic plot of $(A)$ scattering peak position $k_{\max }(t)$ for two mixtures with $c_{\mathrm{d}} / c_{\mathrm{p}}=72$ with asymptotic power-law coarsening with $\varphi=0.12$ and 0.45 and $(C)$ peak height $I_{\max }(t)$ for two mixtures with $c_{\mathrm{c}} / c_{\mathrm{p}}=44$ with asymptotic power-law growth with $\psi=0.069$ and 0.35 .

same region (results not shown). The time evolution of the peak position $k_{\max }$ after the quench is a measure of the coarsening kinetics. In all cases, fast coarsening occurred immediately, and after about $1 \mathrm{~min}$, the coarsening followed power-law scaling, $k_{\max } \propto t^{-\varphi}$, with a coarsening exponent $\varphi$ between 0.06 and 0.47 (see Figure 8A and Table 1). The observed asymptotic power-law dependence of $k_{\max }$ is typical for demixing mixtures in the later stages of nucleation-andgrowth or spinodal decomposition. From Table 1, it may be observed that $\varphi$ is small $(\sim 0.1)$ when $c_{\mathrm{c}}$ is small (less than $5 \mathrm{wt} \%$ ) or when $c_{\mathrm{p}}$ is large (more than $0.15 \mathrm{wt} \%$ ). In these cases, the turbidity increase is also found to be slow $\left(t_{10} \approx\right.$ $400 \mathrm{~s}$ ), corroborating the SALS results. The slow coarsening at high xanthan concentrations is most likely caused by the high continuous phase viscosity (cf. Figure 1), which also slows macroscopic phase separation. Growth rates corresponding to diffusion and coalescence of domains ${ }^{46}$ and, at later times, surface tension driven flow ${ }^{47}$ are predicted to be inversely proportional to the viscosity. At high $c_{\mathrm{c}}$ and low to intermediate $c_{\mathrm{p}}$, we found $\varphi \approx 0.5$, which is comparable to values typically seen experimentally for biopolymer ${ }^{43}$ and colloid-polymer ${ }^{48,49}$ mixtures. For the sample with $c_{\mathrm{c}}=5.52$ and $c_{\mathrm{p}}=0.126 \mathrm{wt} \%\left(c_{\mathrm{c}} / c_{\mathrm{p}}=44\right)$, both the large value of $\varphi$ and the small value of $t_{10}$ found from the turbidity increase point to fast coarsening. The peak height $I_{\max }$ likewise showed asymptotic power-law growth, $I_{\max } \propto t^{\psi}$ (see Figure 8B). The growth exponent $\psi$ (cf. Table 1) was fairly independent of $c_{\mathrm{p}}$ and $c_{\mathrm{c}}$ for samples in which $c_{\mathrm{c}} / c_{\mathrm{p}}<44$ but smaller $(\leq 0.1)$ for samples where $c_{\mathrm{c}} / c_{\mathrm{p}}>44$.

Unlike the microscopy images, the SALS data did not allow discrimination between nucleation-and-growth (NG) and spinodal decomposition (SD). The presence of a peak at nonzero wavevector can be a signature of the dominant density fluctuation developing during SD or of diffusionlimited growth of (interacting) minority phase droplets surrounded by zones depleted in minority component. ${ }^{50-52}$ In the later stages of phase separation, power-law time dependencies of $k_{\max }$ and $I_{\max }$ are expected both for NG and $\mathrm{SD}$. In the case of $\mathrm{NG}$, coarsening occurs through Ostwald ripening of droplets, for which theory ${ }^{53}$ predicts $\varphi=1 / 3$, or droplet coalescence, ${ }^{46}$ for which $\varphi$ is smaller than or equal to $1 / 3$, depending on the collision frequency between droplets. ${ }^{52}$ During SD, domain growth occurs by interfacial and bulk diffusion of the components, leading to a coarsening exponent $\varphi$ between 0.2 and 1.1 depending on the quench depth and the importance of hydrodynamic interactions. ${ }^{54}$ In later stages of SD, the structure breaks up into individual droplets, which grow by Ostwald ripening ${ }^{53,55}$ or coalescence, ${ }^{46}$ so $\varphi=1 / 3$. For concentrated systems, hydrodynamic flow may become the dominant means of mass transport, changing the value of $\varphi$ to $1 .{ }^{47}$ In view of the large range of predicted values for $\varphi$, the likewise large range of experimental values (Table 1) does not allow us to distinguish between NG and SD. The same conclusion applies to the asymptotic growth exponent $\psi$ for the peak height $I_{\max }$. Theories for SD predict $\psi=3 \varphi$ in both the early and late stages of spinodal decomposition. ${ }^{46,47,56}$ For most samples, we find $\psi / \varphi$ values between 2.0 and 4.9 , compatible with the theoretical predictions, but in three cases, we find $\psi / \varphi$ $=0.13-0.81$, significantly smaller than 3. During NG, nucleating droplets of almost final composition grow, so the increase of $I_{\max }$ is caused mainly by an increasing number and size of droplets. For a SD mechanism on the other hand, the increase in $I_{\max }$ is directly related to the density difference between the two coexisting phases because the number of scattering objects is fixed at the start of phase separation.

A distinct feature of SD is that in the initial stage of phase separation only the amplitude of the density fluctuations grows while the length scale remains constant (i.e., $\varphi=$ $0) .57,58$ However, in many demixing systems, this initial phase is very short and the peak position $k_{\max }$ immediately shifts to smaller $k$. The xanthan-PFA systems seem to fall into this category. A distinct feature of $\mathrm{NG}$ is the delay time before a critical nucleus is formed. Again, this is a feature not seen with SALS.

\section{Summary and Conclusions}

This work clearly shows that PFA particles are suitable for studying the kinetics and morphology of phase separation of xanthan-colloid mixtures, even at large concentrations of colloidal particles. The close match of the refractive index of PFA to that of water eliminates multiple scattering complications and permits small angle light scattering (SALS), microscopy, and (depolarized) dynamic light scattering on xanthan-PFA mixtures. Xanthan is nonadsorbing toward PFA, so the observed phase separation is depletioninduced. Because of the small colloid size $(93 \mathrm{~nm})$ relative to the polymer radius of gyration $(264 \mathrm{~nm})$, the systems separate into a colloidal gas and a colloidal liquid phase. 
Optical microscopy enabled us to observe the microstructure of phase-separating samples from early through late stages. In the larger part of the two-phase region phase separation occurred by spinodal decomposition, with coarsening of bicontinuous structures, followed by hydrodynamic flow-induced lane formation. For samples with $\sim 0.1$ wt $\%$ xanthan and $\sim 3 \mathrm{wt} \%$ PFA, close to the binodal, microscopy showed formation of disconnected droplets after an initiation time of ca. $40 \mathrm{~s}$, clearly indicating a nucleation-and-growth mechanism. Although SALS by itself did not allow identification of the mechanisms of coarsening, it does provide quantitative information about coarsening kinetics. For all samples, asymptotic power-law coarsening was observed with exponents compatible with theoretical predictions for both nucleation-and-growth and spinodal decomposition. Both SALS and turbidity measurements demonstrated that large xanthan concentrations resulted in slower coarsening. This corroborates the finding that the macroscopic phase separation rate was smaller at larger xanthan concentrations, most likely because of the high continuous phase viscosity caused by strong overlap of xanthan chains.

Acknowledgment. The authors thank R. H. Tromp and C. G. de Kruif (Nizo Food Research) for providing xanthan, Ausimont for providing PFA spheres, B. M. W. Kuipers and H. J. Klok (Nizo) for help with DLS, and W. Kegel for assistance with optical microscopy. We are grateful to $\mathrm{V}$. Anderson, P. Boulenguer, I. Capron, J. K. G. Dhont, G. A. Vliegenthart, and G.-J. Vroege for helpful discussions. This work was financially supported by The Netherlands Organization for Scientific Research (NWO/Stichting Chemische Wetenschappen).

\section{References and Notes}

(1) Parker, A.; Gunning, P. A.; Ng, K.; Robins, M. M. Food Hydrocolloids 1995, 9, 333-342.

(2) Katzbauer, B. Polym. Degrad. Stab. 1998, 59, 81-84.

(3) Faers, M. A.; Kneebone, G. R. Pestic. Sci. 1999, 55, 312-325.

(4) Gunning, P. A.; Hibberd, D. J.; Howe, A. M.; Robins, M. M. Food Hydrocolloids 1988, 2, 119-129.

(5) Cao, Y.; Dickinson, E.; Wedlock, D. J. Food Hydrocolloids 1990, 4, 185-195.

(6) Luyten, H.; Jonkman, M.; Kloek, W.; Van Vliet, T. In Food colloids and polymers: stability and mechanical properties; Dickinson, E., Walstra, P., Eds.; Royal Society of Chemistry: Cambridge, U.K., 1992.

(7) Hemar, Y.; Tamehana, M.; Munro, P. A.; Singh, H. Food Hydrocolloids 2001, 15, 565-574.

(8) Asakura, S.; Oosawa, F. J. Chem. Phys. 1954, 22, 1255-1256.

(9) Asakura, S.; Oosawa, F. J. Polym. Sci. 1958, 33, 183-192.

(10) Degiorgio, V.; Piazza, R.; Jones, R. B. Phys. Rev. E 1995, 52, 27072717.

(11) Koenderink, G. H.; Philipse, A. P. Langmuir 2000, 16, 5631-5638.

(12) Becker, A.; Katzen, F.; Puehler, A.; Ielpi, L. Appl. Microbiol. Biotechnol. 1998, 50, 145-152.

(13) Jansson, P. E.; Keene, L.; Lindberg, B. Carbohydr. Res. 1975, 45, $275-282$.

(14) Coviello, T.; Kajiwara, K.; Burchard, W.; Dentini, M.; Crescenzi, V. Macromolecules 1986, 19, 2826-2831.

(15) Berth, G.; Dautzenberg, H.; Christensen, B. E.; Harding, S. E.; Rother, G.; Smidsrod, O. Macromolecules 1996, 29, 3491-3498.

(16) Milas, M.; Reed, W. F.; Printz, S. Int. J. Biol. Macromol. 1996, 18, $211-221$
(17) Okuyama, K.; Arnott, S.; Moorhouse, R.; Walkinshaw, M. D.; Atkins, E. D. T.; Wolf-Ullish, C. ACS Symp. Ser. 1980, 141, 411-427.

(18) Capron, I.; Brigand, G.; Muller, G. Polymer 1997, 38, 5289-5295.

(19) Benoit, H.; Doty, P. J. Phys. Chem. 1953, 57, 958-963.

(20) Gamini, A.; Mandel, M. Biopolymers 1994, 34, 783-797.

(21) Büche, F. Physical properties of polymers; Interscience: New York, 1962

(22) Graessley, W. W. J. Chem. Phys. 1967, 47, 1942.

(23) Milas, M.; Rinaudo, M.; Knipper, M.; Schuppiser, J. L. Macromolecules 1990, 23, 2506-2511.

(24) Dhont, J. K. G.; Smits, C.; Lekkerkerker, H. N. W. J. Colloid Interface Sci. 1992, 152, 386-401.

(25) Verhaegh, N. A. M.; Van Duijneveldt, J. S.; Dhont, J. K. G.; Lekkerkerker, H. N. W. Physica A 1996, 230, 409-436.

(26) De Gennes, P. G. C. R. Acad. Sci. Paris B 1979, 288, 359-361.

(27) De Gennes, P.-G. Scaling concepts in polymer physics; Cornell University Press: New York, 1979.

(28) Auserre, D.; Hervet, M.; Rondelez, F. Phys. Rev. Lett. 1985, 54 1948-1951.

(29) Vliegenthart, G. A.; Lekkerkerker, H. N. W. J. Chem. Phys. 2000, $112,5364-5369$.

(30) Poon, W. C. K.; Pirie, A. D.; Pusey, P. N. Faraday Discuss. 1995, 101, 65-76.

(31) Verhaegh, N. A. M.; Asnaghi, D.; Lekkerkerker, H. N. W.; Giglio, M.; Cipelletti, L. Physica A 1997, 242, 104-118.

(32) Poon, W. C. K.; Starrs, L.; Meeker, S. P.; Moussaid, A.; Evans, R. M. L.; Pusey, P. N.; Robins, M. M. Faraday Discuss. 1999, 112, $143-154$.

(33) Tuinier, R.; De Kruif, C. G. J. Colloid Interface Sci. 1999, 218, 201210.

(34) Verhaegh, N. A. M.; Asnaghi, D.; Lekkerkerker, H. N. W. Physica A 1999, 264, 64-74.

(35) Robins, M. M. Curr. Opin. Colloid Interface Sci. 2000, 5, 265272.

(36) Gast, A. P.; Hall, C. K.; Russel, W. B. J. Colloid Interface Sci. 1983, 96, 251-267.

(37) Lekkerkerker, H. N. W.; Poon, W. C. K.; Pusey, P. N.; Stroobants, A.; Warren, P. B. Europhys. Lett. 1992, 20, 559-564.

(38) Leal Calderon, F.; Bibette, J.; Bias, J. Europhys. Lett. 1993, 23, 653659.

(39) Meijer, E.; Frenkel, D. J. Chem. Phys. 1994, 100, 6873-6887.

(40) Ilett, S. M.; Orrock, A.; Poon, W. C. K.; Pusey, P. N. Phys. Rev. E 1995, 51, 1344-1352.

(41) Tanaka, H.; Yokokawa, T.; Abe, H.; Hayashi, T.; Nishi, T. Phys. Rev. Lett. 1990, 65, 3136-3139.

(42) Bodnar, I.; Dhont, J. K. G.; Lekkerkerker, H. N. W. J. Phys. Chem. 1996, 100, 19614-19619.

(43) Tuinier, R.; Dhont, J. K. G.; De Kruif, C. G. Langmuir 2000, 16 , 1497-1507.

(44) Dhont, J. K. G. An introduction to dynamics of colloids; Elsevier: Amsterdam, 1996

(45) Porod, G. In Small-angle X-ray scattering; Glatter, O., Kratky, O., Eds.; Academic Press: London, 1982.

(46) Binder, K.; Stauffer, D. Phys. Rev. Lett. 1974, 33, 1006-1009.

(47) Siggia, E. D. Phys. Rev. A 1979, 20, 595-605.

(48) Rouw, P. W.; Woutersen, A. T. J. M.; Ackerson, B. J.; De Kruif, C. G. Physica A 1989, 156, 876-898.

(49) Penders, M. H. G. M.; Vrij, A. Adv. Colloid Interface Sci. 1991, 36 , $185-217$.

(50) Tromp, R. H.; Jones, R. A. L. Macromolecules 1996, 29, 81098116

(51) Eliçabe, G. E.; Larrondo, H. A.; Williams, R. J. J. Macromolecules 1998, 31, 8173-8182.

(52) Butler, M. F. Biomacromolecules 2002, 3, 676-683.

(53) Lifshitz, I. M.; Slyosov, V. V. J. Phys. Chem. Solids 1961, 19, 3550 .

(54) Dhont, J. K. G. J. Chem. Phys. 1996, 105, 5112-5125.

(55) Wagner, C. Z. Electrochem. 1961, 65, 581-591.

(56) Hashimoto, T.; Itakura, M.; Hasegawa, H. J. Chem. Phys. 1986, 85, 6118.

(57) Cahn, J. W.; Hilliard, J. E. J. Chem. Phys. 1958, 28, 258.

(58) Cahn, J. W. J. Chem. Phys. 1965, 42, 93.

BM025633F 